\title{
WAVELET METHODS FOR CHARACTERISING MONO- AND POLY-FRACTAL NOISE STRUCTURES IN SHORTISH TIME SERIES: AN APPLICATION TO FUNCTIONAL MRI
}

\author{
M.J.Fadilit, E.T.Bullmore and M.Brett \\ * GREYC-ISMRA UMR 6072, Image processing group, 14050 Caen Cedex France \\ ‘ Brain Mapping Unit, Addenbrooke's Hospital, University of Cambridge, CB2 2QQ UK \\ Medical Research Council, Cognition and Brain Unit, Cambridge CB2 2EF UK
}

\begin{abstract}
Functional magnetic resonance imaging (AMRI) time series generally demonstrate serial dependence. This endogenous auto-correlation typically exhibits long-range dependence described by a $\frac{1}{y}$-like power law. In this paper, we present a novel wavelet-based methodology for characterising the noise structure in short-medium length (shortish) fMRI time series. Mono-fractality is assessed in terms of the Hurst exponent and the noise variance. We then investigate potential local stationarity of the Hurst exponent in fMRI data and present a Uniformly Most Powerful test for its time constancy. A novel bootstrap approach is presented as an alternative to the Normal assumption and its advantages are discussed. From several datasets investigated, we specifically show that the $\frac{1}{j}$ model is particularly suited to describe color in fMRI noise. We also demonstrate that even if most of the brain voxels are mono-fractal, there are many locations in the brain where time constancy of the Hurst exponent is violated, i.e., the noise structure is poly-fractal.
\end{abstract}

\section{INTRODUCTION}

Even in the absence of an experimental effect, functional magnetic resonance imaging ( $\mathrm{MRI}$ ) time series generally demonstrate serial dependence. This colored noise or endogenous auto correlation typically has disproportionate spectral power at low frequencies, i.e., its spectrum is $\frac{1}{3}$-like ${ }^{1}$. We have previously investigated the long-range dependence of the noise in AMRI data to make parametric and non-parametric statistical inferences in the wavelet domain [2][3]. This scheme exploits the general whitening or decorrelating property of the orthogonal wavelet transform. For $\frac{1}{f}$-like or fractal noises, e.g., realisations of fractional Gaussian noise (fGin) parameterised by the Hurst exponent $0<I I$. Several sources of color in fMRI have been suggested, including hemonamically-convolved neuronal or instrumental (white) noise, aliased cardiorespiratory pulsation, uncor-

\footnotetext{
'The tem $\frac{1}{f}$-like is used in preference to the simpler $\frac{1}{f}$ partly because many fractional noises are not wide-sense stationary [1].
}

rected head movement, and experimentally-induced variance that is not well-modelled by the design matrix. It is clear from phantom studies that colored noise may arise in the absence of physiological processes and must therefore be due, at least partly, to physical effects [4]. It is also known that the magnitude and form of residual autocorrelation may vary considerably from voxel to voxel within the brain and may be influenced by experimental parameters including the repetition time (TR) between consecutive time points in the series. The characterisation of fMRI noise as a $\frac{1}{7}$-like process does not by itself discriminate between physical and physiological sources. $\frac{1}{j}$-like processes, which have non-integer fractal dimensions in the time domain, are naturally ubiquitous. Physical systems in which many particles are relaxing from excited states at different rates are well known generators of $\frac{1}{7}$-like noise, which seems potentially relevant to fMRI. And physiological time series recorded by electrocardiography and electroencephalography (see [3]) have demonstrated fractal properties, indicating that this may be a common mode of dynamic behavior for human biological processes also. The format of this paper is as follows. We first introduce some notation aspects about orthonormal wavelet transform and long-memory processes. More comprehensive accounts are provided elsewhere. We then describe our local-stationarity test and related aspects such as type-I and type-II error probabilities and the choice of the number of blocks. Finally, these methods are applied to real datasets with and without experimental designs.

\section{WAVELETS AND LONG-MEMORY PROCESSES}

The dyadic orthonormal wavelet transform of a finite energy signal $r(t)$ is defined as the inner product [5]:

$d_{j, k}=\left\langle\int, \psi ; j, k\right\rangle=2^{-j / 2} \int \mathrm{x}(t) \psi\left(2^{-j} t-k\right) d t, \quad(j, k) \in \mathbb{Z}^{2}$

The coefficient $d_{j k}$ is the detail coefficient (or the wavelet coefficient) at scale $j$ and position $k$. The function $\psi$ is the 
wavelet function, whose collection of dilations $j$ and translations $k$ form an orthonormal basis in the Hilbertian space $L^{2}(\mathbb{I t})$. Any continuous function qualifies if the admissibility conditions are satisfied: has a compact support and oscillates $\left(\int v^{\prime}(t) d t=0\right)$. These conditions can be strengthened to include more vanishing moments (up to an order $R$ ) and/or higher order continuous derivatives. From a filterbank point of view, the wavelet $x^{\circ} ; k$ can be viewed as an octave bandpass filter in $\left[-\pi / 2^{j},-\pi / 2^{j+1}\right] \cup\left[\pi / 2^{j+1}, \pi / 2^{j}\right]$. In the present work, for a signal length $\bar{k}$, the indices $(j, k)$ are chosen to be in the set $R=\{j \in\{1, \ldots, J\}, k \in$ $\{0, \ldots, 3 / 2 j-1\}\}$.

In their simplest form, $\frac{1}{i}$ processes are random with a powerlaw spectrum that diverges at the origin as:

$$
S(\delta)_{|j| \sim 0}^{\sim} \frac{t_{\gamma}}{|\delta|^{\gamma}}
$$

Where $\gamma$ is linear function of the Hurst exponent (e.g., $\gamma=$ $2 I I-1$ for an $\mathrm{fG}$ ). Two key properties of the wavelet coefficients of these processes are to crucial for the validity of the mono-fractality test:

P1: Provided $\lambda \geq(\gamma-1) / 2$ the collection of coefficients $d_{j k} \forall k$ are zero-mean stationary, their variances reproduce precisely the power law underlying the scale invariance of the process; i.e. $\operatorname{Var}\left(d_{j k}\right)=\sigma_{i}^{2} 2^{7 j}$

P2: It has been shown that the wavelet coefficients of such a process have a correlation structure whose magnitude decays as $O\left(\left|2 k-2^{\prime} k^{\prime}\right|^{\gamma-1-2 R}\right)[1]$, where $R$ is the number of vanishing moments. Therefore, for $0<H<1$, the residual correlation can be reasonably ignored for any wave let with sufficient number of vanishing moments. Maximum likelihood estimators (MLE) provide an alternative approach to estimation, classification, discrimination or prediction of long-memory or $\frac{1}{t}$ errors and have been developed in the wavelet domain by several authors; see [1] for review. The MLE exists, is unique, efficient and consistent. Moreover, the MLE has a Normal distribution asymptotically which can then be exploited to test the time constancy of the fractal noise parameter $I I$. It can be shown that the MLE of $I$ has a closed-form expression of the Cramer-Rao lower bound which is independent of the true parameter [1]. Thus in the following, we assume that the variance of $\hat{H}$ is known a priori.

\section{PARAMETRIC $I I$ LOCAL STATIONARITY TEST}

The estimation procedure and its statistical performance are the same regardless of the scaling behaviour of the process. Moreover, to an excellent assumption MLE of $I I$ is Gaussian and unbiased with variance that only depends on $J$ and $\lambda$. Estimates taken over adjacent but non-overlapping segments are uncorrelated. These statements were tested statistically via Monte-Carlo (MC) simulations, shown in Fig.1.
It is worth noting that in all simulations in this paper, the data length $\bar{y}$ is deliberately chosen to be smaller than 512 which is in the range of fMRI time series length. The cor-

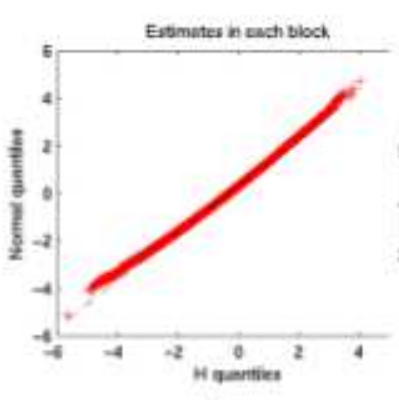

(a)



(b)
Fig. 1. (a) $\hat{I}$ vs Gaussianity. (b) Correlation of the estimates between blocks. For these MC simulations, the following settings are used: $10^{5}$ simulations, $I I=0.8$, $N=2^{9}$, Daubechies $=2$, the data vector was split into 2 to 4 segments.

relation test is derived using a Fisher z-transformation on the correlation coefficient calculated between the estimates from distinct non-overlapping blocks. It is clearly shown from these results that the normal assumption is valid and that the estimates taken from adjacent blocks are indeed uncorrelated. The latter properies are essential which inspire the following local-stationarity test of the Hurst exponent. We suppose that the data is obtained by blending a collection of $n$ mutually independent stationary processes. We estimate $\hat{H}_{i}$ for each segment and the corresponding known variance. Then, testing the time constancy of $I I$ amounts to test whether the collection of mutually independent Gaussian variables $\hat{H}_{i}$ with known variances have the same mean. Therefore, the null hypothesis $H_{0}$ to be tested is $H_{1}=\ldots=H_{\mathrm{r}}=H$ against the altemative that $H_{\mathrm{r}}$ are not all equal. This allows to construct a test with optimal known properties. Particularly, this test is Uniformly Most Powerful Invariant [6] under specific settings where the Normal assumption is valid (i.e., reasonably large number of samples in each block). The set of alternatives can be restricted to include only level shift models.

\subsection{Means homogeneity test}

The problem now is reduced to that of testing the equality of means of $n$ independent variables $H_{i} \sim N\left(\zeta_{i}, \sigma_{i}^{2}\right)$, where $\sigma_{8}^{2}$ are known. The problem of testing $H_{0}$ reduces to a linear hypothesis with known variance through the transformation $H_{i}^{\prime}=H_{i} / \sigma_{i}$, and the UMPI test under suitable group of 
linear transformations rejects $H_{0}$ when:

$$
V=\sum_{i}\left(\frac{H_{i}-\frac{\sum_{i} I_{i} i \sigma_{i}}{\sum_{i} 1 / \sigma_{i}}}{\sigma_{i}}\right)^{2}>C
$$

where $C$ is the critical level determined by $\int_{C}^{x} \chi_{n-1}^{2}\left(v^{\prime}\right) d t^{\prime}=$ $\alpha$, and $\alpha$ is the significance level. The non-centrality parameter $\delta$ can be calculated replacing $H_{i}$ by $\zeta_{i}$ in (3).

\subsection{Statistical properties}

The statistical properties of the wavelet-based time constancy test of $I I$ is tightly related to the Normality and independence assumptions made along the paper. The Normality is clearly violated for short-medium (shortish) time series, a situation encountered in practice (e.g., routinely in fMRI only hundred of data points are collected by the experimenter). But how would the mon-fractality test behave under these circumstances? The other major issue is the prior choice of the number of segments $\eta$, which is a crucial step in the algorithm.

Type I error control It is clear that for small samples, the parametric test will fail to control the significance level such that the actual probability of type-I error equals the nominal error rate. This has been checked numerically a posteriori using MC simulations. 1000 512-dimensional fGn sample paths were synthesised with constant $I=0.8$. Each vector was split into $n$ segments. Counting the number of times out of $10^{3}$ that $V$ fell in the critical region with a nominal significance level of $100 \times(1-\alpha)=95 \%$ gives the observed type-I error. It is clear that for high $\%$, the number of available samples in each segment decreases and the Normal assumption is no longer valid. In addition, the effect of residual correlation between wavelet coefficents tends to increase with small samples, and then affects the type-I error probability. This is evidenced from simulations in Fig.2, where the test systematically results in an inflated or uncontrolled type-I error.

It is known that the use of bootstrap is tied to the concurrent availability of the Normal assumption. Then, to overcome this major limitation, a bootstrap based inference approach can be used. We outline here its main steps as this method will be detailed in a forthcoming paper. Exploiting the whitening property $\mathrm{P} 2$ of the orthornormal wavelet transform and property $\mathrm{P} 1$, one can resample with replacement the appropriately rescaled detail coefficients $d_{j k} /\left[\hat{\sigma}_{i}{ }^{2} 2^{i j}\right]$ in each block. This gives the resampled bootstrap residuals $\epsilon_{j k}^{*}$. Under $H_{0}, H_{i}$ are all equal to $H$, and then the mean $\hat{n}=\frac{\sum_{i} N_{i} H_{i}}{\sum_{i} N_{i}}=I I$ under $I_{0}$. Thus, we reconstruct the bootstrap resamples $x^{* *}$ as the inverse wavelet transform of the coefficients $2^{\bar{j} j} \epsilon_{j k}^{*}$. The bootstrap statistic $V^{-*}$ is calculated replacing $I_{\gamma}$ by their bootstrap versions [7]. We have a bootstrap method which assumes that $H_{0}$ is true. This procedure guaranties that $\mathrm{I}^{-*}$ mimicks the distribution of $\mathrm{V}$ under $I_{0}$. The test that rejects $I_{0}$ if $V$ is larger than the $1-\alpha$-quantile of the conditional distribution of $V^{-*}$ asymptotically has the level $\alpha$, with a higher correc tness order than the Normal assumption. This assertion has to be proved and is under current investigation. When this inference procedure is used instead of the Normal assumption, the observed type-I error probability is excellent over the full range of probability thresholds (see Fig.2). For high values of $\eta$, the bootstrap method tends to be slightly conservative.
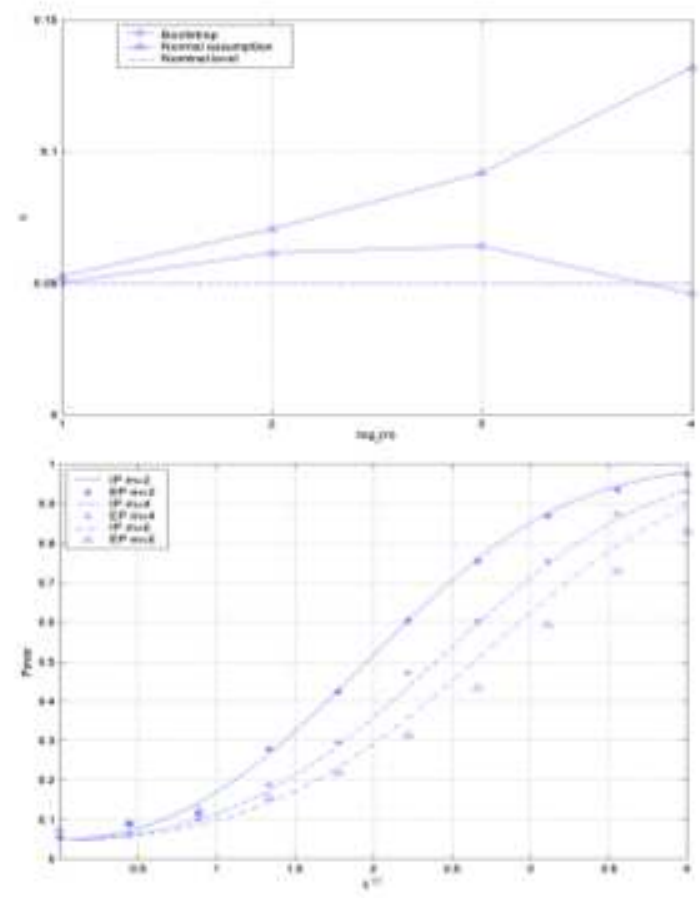

Fig. 2. Statistical properties of the Hurst exponent localstationarity test. Top: Confidence level. Bottom: Power of the test. MC simulations settings: $\alpha$ nominal level $=0.0 \bar{s}$, $10^{5}$ simulations, $I I=0.8, \lambda=2^{9}$, Daubechies $=2$, the data vector was split into 2 segments.

Power of the test For this analysis, a simple level shift $\Delta I I$ is applied at $\hat{N} / 2$, keeping the other simulations settings as stated in Fig.2. The ideal power (IP) functions assuming non-central $\chi_{n-1}^{2}$ under $H_{1}$ are plotted with lines. The empirical power (EP) functions are plotted with symbols. It is clearly shown that for reasonable number of samples (c.g., 512), the observed power is close but underestimated by the test under Normal assumption. The difference is still acceptable and allows to say that the type-II error probability is controlled in a reasonable range by this test. Comparison to the bootstrap test under $I_{1}$ needs also to be performed in the future. 
The effect of the number of segments The choice of the number of blocks is subject to the compromise between power and time resolution to track fine changes in the Hurst exponent with sufficient power. On the one hand, decreasing $n$ increases the variance in the estimate $\hat{I}$ proportionally to r. This increases uncertainty on the test decision and therefore yields a power degradation. This assertion is confirmed by simulations in Fig.2. Consequently a limit on the smallest value of $r$ can therefore be fixed for a given required power. Another practical issue encountered on this inferior limit is the minimum number of octave bands required for the estimation algorithm. Thus, for any given wavelet with a support length $L_{0}$, the smallest scale must satisfy $J_{\lim }=\log _{2}\left(\frac{N}{r \mathcal{L}_{\mathrm{C}}}\right) \geq 2$. On the other hand, decreasing $n$ coarsens the temporal resolution of the test and so does not allow us to track fine changes in $I I$ over time. A limit can be imposed by the stationarity bandwith that can be estimated from the signal [5]. Another approach would exploit the unconditional adaptive time axis segmentation by an appropriate Coifman-Meyer cosine packets basis, where the covariance of the locally-stationary process is almost diagonal [5]. The latter alternatives are areas of future research.

\section{APPLICATION TO FMRI DATA}

These wavelet-based methods to characterise the fractality of noise structures in shortish time series were applied to fMRI datasets acquired with and without experimental stimulation. Several datasets were investigated from two MR scanners ( $101.5 \mathrm{~T}$ and $63 \mathrm{~T}$ datasets), and with different acquisition parameters settings. Our findings were consistent with previous results [4]. Fig. 3 shows an example for a single subject $3 \mathrm{~T}$ dataset $(T R=1 s, \lambda=312$, no experimental paradigm). It is shown from $I I$ maps that for most voxels, the noise structure is clearly fractal with disproportionate $\frac{1}{f}$ power law. This is confirmed by the goodness of fit measure (GOF) based on the likelihood score. The noise variance is not homogeneously distributed but tends to be larger in outer (cortical) areas of the brain. These results were reproducible across all the datasets investigated even for phantoms, demonstrating that instrumental effects are partly responsible for the long-range dependence in the noise. Nevertheless, the $\frac{1}{7}$ model can be inappropriate in some locations of the brain as revealed by the GOF measure, suggesting that the Hurst exponent might vary across time. Then, the local-stationarity test was applied to these voxels $(n=6)$. By inspection of Fig. 3 it seems that though many voxels are $\frac{1}{y}$-like, only cortical voxels are likely to refute the $I_{0}$ of monofractality. This suggests that though some colored noise may be of instrumental origin, particularly complex poly-fractal noise is most likely in cortical voxels. This also points to the fact that time constancy of $I I$ and stationarity in general could be a gross assumption

particularly at these locations of the brain.

\section{CONCLUSION}

We presented a wavelet-based methodology for characterising the noise structure in time series with an application to fMRI. We also proposed a novel test for local-stationarity of the Hurst exponent and showed the superionity of the bootstrap based inference approach over the Normal assumption. The preliminary observations on $\mathrm{AMRl}$ are very encouraging and indicate that the methodology is feasible for fMRI data analysis and may offer considerable advantages over alternative estimators given the prevalence of long-memory errors in these data.
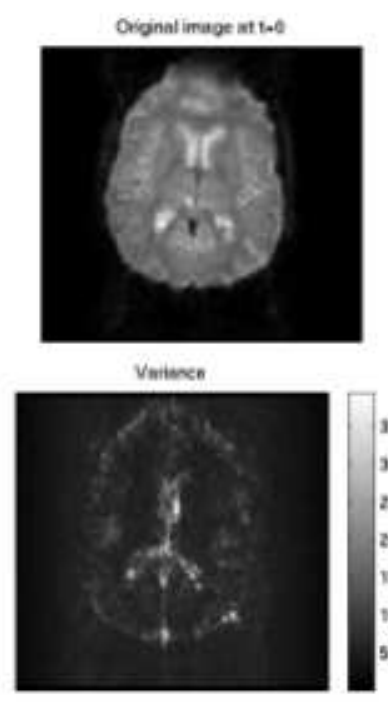

Fig. 3. Results for a single subject 3T dataset.

\section{REFERENCES}

[1] G.W. Wornell, Signal processing with fractals: a waveletbased approach, Prentice Hall, 1996.

[2] M.J.Fadili and E.T.Bullmore, "An approximate wavelet-based mle of regression models with long-memory errors," IEEE Trans. Sig. Proc., 2000 (Submitted).

[3] E.T.Bullmore C.Long J.Suckling M.J.Fadili et al., "Color noise and computational inference in time series analysis: Resampling methods in time and wavelet domains," Human Brain Mapping, vol. 12, no. 2, pp. 61-78, Feb. 2001.

[4] E.Zarahn G.K.Aguire and M.D'Esposito, "Empirical analyses of bold finri statistics: I. spatially unsmoothed data collected under null hypothesis conditions," Neurolmage, vol. 5, pp. $179-197,1997$.

[5] S.G.Mallat, A Wavelet tour of signal processing: 2nd Edition, Academic Press, London, 1999.

[6] E. Lehmann, Testing statistical hypothesis, Wiley, 1959.

[7] P.Hall, The Bootstrap and Edgenorth expansion, Springer, New York, 1992. 